BI-TP 2004/09

CERN-PH-TH/2004-055

EPFL-ITP-LPPC/2004-1

HIP-2004-14/TH

hep-ph/0404058

\title{
Effective gauge theories on domain walls via bulk confinement?
}

\author{
M. Laine $^{\mathrm{a}}$, H.B. Meyer ${ }^{\mathrm{b}}$, K. Rummukainen ${ }^{\mathrm{c}, \mathrm{d}, \mathrm{e}}$, M. Shaposhnikov ${ }^{\mathrm{f}}$ \\ ${ }^{a}$ Faculty of Physics, University of Bielefeld, D-33501 Bielefeld, Germany \\ b Theoretical Physics, University of Oxford, 1 Keble Road, Oxford, OX1 3NP, UK \\ ${ }^{\mathrm{c}}$ Department of Physics, Theory Division, CERN, CH-1211 Geneva 23, Switzerland \\ ${ }^{\mathrm{d}}$ Department of Physics, University of Oulu, P.O.Box 3000, FIN-90014 Oulu, Finland \\ ${ }^{\mathrm{e}}$ Helsinki Institute of Physics, P.O.Box 64, FIN-00014 University of Helsinki, Finland \\ ${ }^{\mathrm{f}}$ Institute of Theoretical Physics, Swiss Federal Institute of Technology (EPFL), \\ BSP-Dorigny, CH-1015 Lausanne, Switzerland
}

\begin{abstract}
We study with lattice techniques the localisation of gauge fields on domain wall defects in $2+1$ dimensions, following a scenario originally proposed by Dvali and Shifman for $3+1$ dimensions, based on confining dynamics in the bulk. We find that a localised gauge zeromode does exist, if the domain wall is wide enough compared with the confinement scale in the bulk. The range of applicability of the corresponding low-energy effective theory is determined by the mass gap to the higher modes. For a wide domain wall, this mass gap is set by "Kaluza-Klein modes" as determined by the width. It is pointed out that in this regime the dynamical energy scales generated by the interactions of the localised zero-modes are in fact higher than the mass gap. Therefore, at least in $2+1$ dimensions, the zero-modes alone do not form a low-energy effective gauge theory of a traditional type. Finally, we discuss how the situation is expected to change in going to $3+1$ dimensions.
\end{abstract}

April 2004 


\section{Introduction}

In brane-world scenarios with infinite [1, 2, 3] or large [4 extra dimensions, it is assumed that all the fields of the Standard Model have wave functions that are localised in transverse directions, making the physics to be four-dimensional (4d) at small energy scales. The field-theoretical realisation of branes is related to topological defects - stable solutions of the classical equations of motion, which depend on the transverse coordinates only. In five dimensions, the necessary defect is a domain wall, in six dimensions it is a (4d) string, in seven dimensions a monopole, etc. (For an explicit construction of these solutions in the case of localisation of gravity, for instance, see Refs. [5, 6, 7].)

Small perturbations of the fields around the topological defect solutions may form a lowdimensional effective theory - potentially the Standard Model (for a general discussion of perturbations see, e.g., Ref. [8]). Apart from the requirement that the wave function of the "fundamental" (or lowest-energy) mode be centered around the brane in transverse directions, such that it has long-wavelength perturbations in four directions only, a successful localisation poses other constraints as well. Indeed, either the perturbations of all the higher modes should be separated from those of the fundamental mode by a sufficient mass gap, in order not to be observable in low-energy experiments, or, if there is no mass gap, the perturbations of the higher modes should interact very weakly with those of the fundamental one.

The possible solutions to these requirements can roughly be divided into two classes (for a recent review, see Ref. [9]). On one hand there are mechanisms which, in one way or the other, invoke effects related to gravity (such as a "warped" metric [10, 3]). In this way scalars [11, 12, fermions 11, 13]-16], Abelian gauge fields [12, 14, [16]-19] and gravity [3] can be localised on a brane, although the construction of the full Standard Model is still far from being achieved. On the other hand, there are mechanisms which work in flat spacetime and are purely field theoretic in origin. For example, the localisation of fermions on a brane may be due to the presence of fermionic zero-modes on topological defects [1, 20, while scalars can be attached to the brane through their interactions with the field forming the topological defect (and the perturbations of the defect forming field itself constitute a light scalar field).

The most difficult problem along these lines seems to be the localisation of massless nonAbelian gauge fields (for a general discussion, see Ref. [21]). If the mechanism related to gravity is used, for instance, then for typical warp factors, the bulk gauge fields have a spectrum which is not separated by a mass gap from the localised modes, so that no effective field theory can be constructed. ${ }^{1}$ At the same time, in the case without gravity, no perturbative mechanism of gauge field localisation is available at present, as far as we know. ${ }^{2}$

A very interesting non-perturbative purely field theoretic idea for gauge field localisation

\footnotetext{
${ }^{1}$ Both massless and massive vector fields can be localised on a brane, such that the fundamental mode $i s$ separated from the higher ones by a non-zero mass gap, if the warp factor is tuned appropriately "by hand" [22] 23] (see also Ref. [24).

${ }^{2}$ Although of significant interest, we do not consider mechanisms related to a high degree of unbroken supersymmetry in this paper (see, e.g., Ref. [25] and references therein).
} 
was put forward some time ago by Dvali and Shifman [26]. It uses the fact that non-Abelian gauge theories are strongly coupled in the infrared and that a mass gap can be generated for the bulk modes by confinement effects. In short: consider a confining theory in the bulk, based on some group G. Construct a topological defect that "eats up" the necessary number of dimensions and breaks this symmetry down to $\mathrm{G}^{\prime}$ inside the defect, while leaves it intact outside. Then the gauge fields related to $\mathrm{G}^{\prime}$ are localised on the defect and are separated by a mass gap from the bulk modes, which are massive because of confinement. The resulting low-energy effective theory is thus a four-dimensional gauge theory based on $\mathrm{G}^{\prime}$.

Of course, to use this idea for the construction of a realistic theory, one would first have to find a confining gauge theory in $4+n$ dimensions, with $n \geq 1$. Non-Abelian gauge theories of the usual type are, however, not renormalisable when extra dimensions are involved. In five dimensions, for instance, lattice simulations [27] do not reveal any second order phase transition that can be used for a continuum formulation of a confining theory. ${ }^{3}$ Thus, to have a simple renormalisable framework, Dvali and Shifman assumed the bulk dynamics to be that of $4 \mathrm{~d}$ gauge theory, so that the low-energy dynamics is that of three-dimensional (3d) gauge theory.

Even if plausible after this simplification, the idea does still involve some untested assumptions. First of all, it may not be immediately clear why the non-perturbative confinement effects, acting in the bulk and admittedly suppressed on the brane, could not generate an effective mass term for the brane gauge fields; this depends after all on the precise boundary conditions that the bulk phase poses on the localised modes [29]. Another question, necessary for understanding whether the effective theory is truly lower dimensional than the bulk theory, is related to the magnitudes of the confinement induced mass gap $M_{c}$, the typical energy scale of the low-dimensional theory $m_{l}$, and the masses of higher "Kaluza-Klein" (KK) excitations $M_{k}$, localised on the brane. ${ }^{4}$ To hide extra dimensions at small energies, the scale $m_{l}$ must be much smaller than the masses of particles in the bulk as well as those of the KK-excitations: $m_{l} \ll M_{k}, m_{l} \ll M_{c}$. Otherwise, the effects of higher dimensions are not suppressed. This point has not been discussed in Refs. 26, 29, as far as we can judge.

Given the open ends, it is the purpose of this paper to test the idea of Ref. [26] with simple non-perturbative lattice simulations. We consider $\mathrm{SU}(2)$ gauge theory coupled to an adjoint scalar field with an effective space-dependent mass parameter induced by the kink solution, as in the original proposal [26]. Since going to higher dimensions leads us to a shaky ground we will deal, following Ref. [26], with renormalisable theories only, i.e. consider at most a 4d bulk. For better numerical resolution and since the physics arguments are almost unchanged (see below), we will however reduce the dimensionality to be three in total, such that the low-energy effective theory is supposed to be two-dimensional (2d).

Our lattice simulations do support the Dvali-Shifman conjecture on the existence of a gauge

\footnotetext{
${ }^{3}$ For recent discussions and references see, e.g., Refs. 28.

${ }^{4}$ Even though there is no compact dimension involved, we refer to the tower of localised states above the fundamental mode as Kaluza-Klein excitations, due to the fact that they have a similar spectrum.
} 
field localised on the brane. In other words, if the dynamics of the theory is probed with external sources separated by large distances along a (wide enough) brane, a force specific to a massless localised gauge mode will indeed manifest itself. We find, however, that in this limit there is no hierarchy of (dynamical) scales between the $2 \mathrm{~d}$ localised mode and certain higher excitations and, therefore, that the low-energy theory cannot be considered to be a traditional $2 \mathrm{~d}$ gauge theory.

This paper is organised as follows. In Sec. 2 we review the basic mechanism as proposed by Dvali and Shifman [26]. In Sec. 3 we derive its signatures for a specific observable, the static force between two heavy test charges living on the brane, and in Sec. 4 discuss how the signatures change if the world-volume is finite. In Sec. [5 the system is put on the lattice and the observables are written in a form accessible to Monte Carlo simulations. The main results are presented in Sec. [6] and our conclusions as well as a brief outlook, in Sec. [7.

\section{Overview of the Dvali-Shifman mechanism}

The model suggested by Dvali and Shifman has two scalar fields, a gauge singlet $\eta$ and an adjoint scalar $\chi$, and has the following Euclidean action in the case of a $3 \mathrm{~d}$ bulk:

$$
\begin{aligned}
S_{\mathrm{E}} & \equiv \int \mathrm{d}^{3} x \mathcal{L}_{\mathrm{E}}, \\
\mathcal{L}_{\mathrm{E}} & \equiv \frac{1}{2} \operatorname{Tr} F_{k l}^{2}+\operatorname{Tr}\left[D_{k}, \chi\right]^{2}+\lambda \operatorname{Tr}\left[\chi^{2}\right]^{2}+\gamma \operatorname{Tr}\left[\chi^{2}\right]\left(u^{2}-v^{2}+\eta^{2}\right) \\
& +\frac{1}{2}\left(\partial_{\mu} \eta\right)^{2}+\frac{1}{4} \kappa\left(\eta^{2}-v^{2}\right)^{2},
\end{aligned}
$$

where $k, l=1, \ldots, 3, D_{k}=\partial_{k}+i g A_{k}, A_{k}=A_{k}^{a} T^{a}, \chi=\chi^{a} T^{a}, F_{k l}=(1 / i g)\left[D_{k}, D_{l}\right]$, and $T^{a}$ are the Hermitean generators of $\mathrm{SU}\left(N_{c}\right)$, normalised as $\operatorname{Tr}\left[T^{a} T^{b}\right]=\delta^{a b} / 2$. Summation over repeated indices is understood. The coupling constants $\lambda, \gamma, \kappa$ and the parameters $u^{2}$ and $v^{2}$ are assumed to be positive, and $v^{2}>u^{2}$.

The classical vacuum of the theory is at $\chi=0, \eta= \pm v$ and the perturbative spectrum consists of the scalar singlet with the mass $m_{\eta}^{2}=2 \kappa v^{2}$, the scalar triplet with the mass $m_{\chi}^{2}=\gamma u^{2}$ and massless gauge bosons corresponding to the gauge group $\mathrm{SU}\left(N_{c}\right)$. Because of strong coupling in the infrared, the vector boson spectrum aquires a mass gap of the order of the $3 \mathrm{~d}$ confinement scale, $\Lambda \sim g^{2}$. In other words, gluons form bound states - glueballs with a mass of the order of $\Lambda$. Depending on the relation between $\Lambda$ and $m_{\chi}$, bound states of the triplet scalar and gluons can have masses $\mathcal{O}(\Lambda)$ or $\mathcal{O}\left(m_{\chi}\right)$.

Now, the model of Eq. (2.2) always has a kink solution, $\chi=0, \eta(z)=v \tanh \left(\frac{m_{\eta} z}{2}\right)$. By inspecting whether the fluctuation Hamiltonian around this solution develops a negative eigenvalue, it is seen that the solution is unstable against $\chi$-field condensation near $z=0$, provided that

$$
\gamma v^{2}>m_{\chi}^{2}+\frac{1}{2} m_{\chi} m_{\eta}
$$


In this case the stable classical solution contains a non-zero $\chi$ field as well, and has the asymptotics $\chi_{3} \rightarrow 0, \eta \rightarrow \pm v$ at $z \rightarrow \pm \infty$, whereas the components $\chi_{1}$ and $\chi_{2}$ can be chosen to be zero. For a general choice of parameters the explicit solution can easily be constructed numerically. The analytic form can be found for a specific choice of parameters, namely for

$$
2 \gamma u^{2}=\frac{\kappa \lambda-\gamma^{2}}{\lambda-\gamma} v^{2}
$$

and is given by

$$
\eta(z)=v \tanh \left(m_{\chi} z\right), \quad \chi_{3}(z)=\sqrt{\frac{\kappa-\gamma}{\gamma-\lambda}} \frac{v}{\cosh \left(m_{\chi} z\right)} .
$$

In the special case $\kappa=\gamma=\lambda, \eta(z)$ remains the same while

$$
\chi_{3}(z)=\frac{\sqrt{v^{2}-2 u^{2}}}{\cosh \left(m_{\chi} z\right)} .
$$

To get the mechanism to work, the following choice of parameters is proposed. Inside the defect the $\mathrm{SU}\left(N_{c}\right)$ symmetry is partially broken to $\mathrm{G}^{\prime}$, and the masses of the corresponding vector bosons are assumed to be large compared with the confinement scale $\Lambda$,

$$
\frac{\gamma\left(v^{2}-u^{2}\right)}{\lambda} \gg g^{2}
$$

At the same time, the width of the domain wall is chosen to be larger than the inverse of the confinement scale, $g^{2} \gg m_{\chi}$. These two requirements suppress the non-perturbative confinement effects inside the defect. Together with Eq. (2.3), they pose restrictions on the parameter space, but given that there are several parameters at our disposal $(u, v, \lambda, \gamma, \kappa)$, all the requirements can easily be satisfied simultaneously.

At the quadratic level the spectrum of perturbations around the domain wall contains a few normalizable localised scalar modes (there is one zero-mode associated with brane translations and fluctuations), a continuous spectrum of scalar excitations that starts from $\min \left(m_{\eta}, m_{\chi}\right)$, and a gapless continuous spectrum of vector excitations corresponding to the unbroken group $\mathrm{G}^{\prime}$. Now, because the true non-perturbative spectrum of bulk gauge excitations is massive, it was conjectured in Ref. 26] that the vector bosons of the unbroken group cannot escape the brane and thus the true spectrum of gauge excitations around the domain wall does contain normalisable vector modes related to $G^{\prime}$. If true, the low-energy effective theory is just a $2 \mathrm{~d}$ gauge theory in our case.

Further support for the Dvali-Shifman idea has been provided by Arkani-Hamed and Schmaltz, who argued [29, based on the 't Hooft - Mandelstam picture of confinement, that the bulk acts as a Neumann boundary condition for the gauge fields corresponding to $\mathrm{G}^{\prime}$, such that there is a true localised zero-mode, unlike in the case of a Dirichlet boundary condition, whereby a zero-mode is excluded. ${ }^{5}$ Since the physics of confinement is involved,

\footnotetext{
${ }^{5}$ For further work on the topic see, e.g., Ref. 30.
} 
however, a non-perturbative check of the mechanism would be welcome, and this is one of the aims of the present work. Another problem, related to mass gaps and the dimensionality of the low-energy theory, has been mentioned already in the Introduction, and will be explained in more detail below.

\section{How to probe the properties of the low-energy effective theory?}

Since our main interest here is in the gauge fields, we will ignore in the following all the dynamics related to the scalar singlet field $\eta$. To achieve this we go to the rest frame of the domain wall, and also treat it as infinitely rigid, which means that we remove the $3 \mathrm{~d}$ zero-mode related to translations and fluctuations of the domain wall.

The remaining dynamical degrees of freedom constitute the $\mathrm{SU}\left(N_{c}\right)$ gauge + adjoint Higgs theory, formally defined by the action

$$
\begin{aligned}
S_{\mathrm{E}} & \equiv \int \mathrm{d}^{3} x \mathcal{L}_{\mathrm{E}}, \\
\mathcal{L}_{\mathrm{E}} & \equiv \frac{1}{2} \operatorname{Tr} F_{k l}^{2}+\operatorname{Tr}\left[D_{k}, \chi\right]^{2}+m^{2}(z) \operatorname{Tr}\left[\chi^{2}\right]+\lambda\left(\operatorname{Tr}\left[\chi^{2}\right]\right)^{2},
\end{aligned}
$$

where $m^{2}(z)$ is simply some profile for the mass of $\chi$, which we fix "by hand" to be

$$
m^{2}(z) \equiv m_{2}^{2}+\frac{m_{1}^{2}-m_{2}^{2}}{\cosh ^{2}(z / \ell)}
$$

where, in the notation of Sec. $2 m_{2}^{2} \equiv m_{\chi}^{2}=\gamma u^{2}$ represents the mass of the scalar triplet outside the brane, while $m_{1}^{2}=\gamma\left(u^{2}-v^{2}\right)$ is negative and is related to the mass of the scalar boson inside the brane. As already discussed (cf. Eq. (2.7)), we assume that the masses of the vector bosons inside the brane are large, $m_{W}^{2} \sim-g^{2} m_{1}^{2} / \lambda \gg g^{4}$. The domain wall width, $\ell$, is for generality now treated as a parameter independent of $m_{\chi}$, and is supposed to be large enough,

$$
\ell \gg \frac{1}{g^{2}}
$$

to suppress the influence of bulk confinement on the localised gauge field. In addition, to have condensation of $\chi$ inside the brane, we must require (cf. Eq. (2.3)) that $m_{1}^{2} \lesssim-m_{2} / \ell$.

The dimensionless combinations of the parameters, determining the actual dynamics, can be chosen as

$$
\alpha \equiv \ell g^{2}, \quad x \equiv \frac{\lambda}{g^{2}}, \quad y_{1,2} \equiv \frac{m_{1,2}^{2}}{g^{4}},
$$

where the mass parameters (the only ones requiring renormalisation in three dimensions) are for convenience assumed to be evaluated in the $\overline{\mathrm{MS}}$ scheme at the scale $\bar{\mu}=g^{2}$.

Let us assume now that the Dvali-Shifman conjecture is correct and estimate the parameters of the localised gauge theory. Since the wave function of the massless mode is localised on 
a length of the order of the width of the domain wall, the $2 \mathrm{~d}$ effective gauge coupling $g_{2}$ is simply

$$
\frac{1}{g_{2}^{2}} \sim \frac{\ell}{g^{2}}
$$

If two opposite static test charges are put on the brane, one would then expect that they are attracted, at large distances (to be specified presently) with a force $F \sim g_{2}^{2}$ that does not depend on the distance. Note that this holds also for $\mathrm{G}^{\prime}=\mathrm{U}(1)$, since the Coulomb potential is linear in $1+1$ dimensions.

Besides the massless mode one would expect to also have a whole tower of states with the same quantum numbers and with an energy spacing of the order of $E \sim 1 / \ell$, with $E$ coming simply from the uncertainty principle. These states are not seen at distances $r \gg \ell$ or at energies $E \ll 1 / \ell$, so that the force derived above should be valid for $r \gg \ell$. For $m_{W}^{-1} \ll r \ll \ell$ the 2 d Coulomb law is expected to be replaced by the 3 d Coulomb law with the force $F \sim g^{2} / r$, while at even smaller distances the massive $W$ will contribute as well, changing the numerical coefficient in front of the $1 / r$-dependence of the force.

If, on the contrary, the localised zero-mode acquires a mass $m_{\gamma}$ due to interactions with the bulk modes, the force between the test charges at $r \gg \ell$ will have a Yukawa character, $F \sim g_{2}^{2} \exp \left(-m_{\gamma} r\right)$, whereas for smaller $r$ the behaviour is still as described above.

These two different behaviours of the force at $r \gg \ell$ can be distinguished in lattice simulations, as will be described below, so that a conclusion can be reached on the existence of a localised vector zero-mode. As we will see, the presence of a vector zero-mode is confirmed, and the dependence of the $2 \mathrm{~d}$ coupling constant on the width of the domain wall (Eq. (3.6)) is also found to be as expected.

Let us now discuss whether the low-energy theory can indeed be considered to be $1+1$ dimensional electrodynamics. If so, the typical energy scale $E_{0}$ of the $1+1$ dimensional theory must be smaller than the mass gap to the first excited KK mode. To be concrete, let us imagine adding fermions to the $3 \mathrm{~d}$ theory and let them interact with the singlet field in a way that ensures the existence of fermionic zero-modes. The gauge coupling of these fermions is $g_{2}$. Then, the theory of the zero-modes (fermionic and vector) is simply the Schwinger model, the solution of which tells that bosonic scalar states with the mass $E_{0} \sim g_{2}$ are formed. The KK tower of states decouples, provided that $E_{0} \ll 1 / \ell$. This inequality, together with Eq. (3.6) and the estimate $E_{0} \sim g_{2}$, gives $\ell \ll g^{2}$, which is in contradiction with the initial assumption of Eq. (3.4). In other words, we do not expect the low-energy effective theory to be the Schwinger model, it will rather be a more complicated $1+1$ dimensional theory incorporating not only the massless mode but also its KK excitations.

This expectation can also be tested on the lattice. Indeed, if for some reason the mass gap to the KK excitations is considerably higher than $1 / \ell$ and corresponds to some distance scale $\ell_{0} \ll \ell$, then the $1+1$ dimensional Coulomb law will be valid to considerably smaller distances. As we will see in the lattice simulations, the deviations start really from $r \sim \ell$, and are characterised by a mass gap $\sim 1 / \ell$. Therefore, the low-energy theory, though giving 
an apparently $1+1$ dimensional force at the distance scales $\gg \ell$, is in general not that of the zero-modes alone.

We also note that the other logical possibility, $\ell<1 / g^{2}$, does not lead to $1+1$ dimensional electrodynamics either. In this case the spread of the potentially massless localised mode is given by the inverse confinement scale, $1 / g^{2}$ in our case. Thus, the effective $2 \mathrm{~d}$ gauge coupling is $g_{2}^{2} \sim g^{4}$, and, therefore, the typical $2 \mathrm{~d}$ energy scale is of the same order as the mass gap to the bulk modes.

To conclude this section, we note that the specific line of reasoning above is related to the case of $3 \rightarrow 2$ compactification. In the last section we however provide arguments that the pattern is essentially the same also for $4 \rightarrow 3$ compactification, in the generalised case that $\mathrm{G}^{\prime}$ is a non-Abelian group. Unfortunately, we have nothing to say about (the most interesting) higher dimensional case.

\section{Finite-size scaling of the Abelian static force in a periodic box}

As explained in the previous section, the probe to be used in order to test the conjecture, is the static force between infinitely heavy test charges living on the brane. The motivation for this choice is that theoretical predictions, as reviewed in the previous section, are unambiguous, and that lattice measurements, as discussed in Sec. 5. can be made rather precise, employing recent technical advances [31]. To perform the lattice simulations, periodic boundary conditions will be used, so we shall first discuss how this changes the expectations for the static force presented above for the infinite-volume case.

To fix the notation, let us consider the three-dimensional volume to be a Euclidean hypertorus (or box with periodic boundary conditions in all directions), with a coordinate $r$ in the spatial direction along the brane, $t$ in the temporal direction along the brane, and $z$ along the "bulk", perpendicular to the brane. The extents of each direction (if finite) are denoted by $L_{r}, L_{t}, L_{z}$, respectively. The brane is located at $z=0$.

The static force is defined in the usual way. We introduce a rectangular Wilson loop $W(R, T ; z)$ in the $(r, t)$-plane, at some fixed $z$, of size $R \times T$. The force $F(R ; z)$ is defined through the potential $V(R ; z)$ as

$$
F(R ; z)=-\frac{\mathrm{d} V(R ; z)}{\mathrm{d} R}=\lim _{T \rightarrow \infty} \frac{1}{T} \frac{\mathrm{d}}{\mathrm{d} R} \ln W(R, T ; z),
$$

where we assumed that $L_{t}=\infty$.

If we imagine for a moment that the system is homogeneous and perturbative, a leading order computation in $g^{2}$ gives

$$
F(R ; z)=g^{2} C_{F} \frac{1}{L_{z}} \sum_{p_{z}} \frac{\sinh \left[p_{z}\left(L_{r} / 2-R\right)\right]}{2 \sinh \left(p_{z} L_{r} / 2\right)},
$$


where $C_{F}$ is the quadratic Casimir of the fundamental representation, $C_{F} \equiv\left(N_{c}^{2}-1\right) / 2 N_{c}$, and $p_{z}=2 \pi n / L_{z}$, with $n$ an integer. This result is exact for an Abelian theory (Coulomb law), but in a non-Abelian confining theory it is only valid at short distances and, in general, the periodicity it displays will be lost at large distances. Nevertheless, as we will see, various interesting limits can be obtained from this simple expression. In the following we set $N_{c}=2$ as in the actual simulations, so that $C_{F}=3 / 4$.

Let us consider three limiting cases:

Infinitely thin brane: the symmetric phase. In the confining symmetric phase, the force is analytically computable only at small distances (the Coulomb part). Because of the mass gap, we can set $L_{z} \rightarrow \infty$ in Eq. (4.2), so that the sum $\sum_{p_{z}}$ becomes an integral. Then,

$$
\begin{aligned}
F(R ; z) & =\frac{3}{4} \frac{g^{2}}{2 \pi R}, & & R \ll\left(g^{2} C_{A}\right)^{-1}, \\
& =c_{1}\left(g^{2} C_{A}\right)^{2}, & & \left(g^{2} C_{A}\right)^{-1} \ll R \ll L_{r} / 2,
\end{aligned}
$$

where $\sigma=c_{1}\left(g^{2} C_{A}\right)^{2}$ is the string tension of $3 \mathrm{~d} \mathrm{SU}(2)$ Yang-Mills theory with adjoint matter, and $C_{A}=N_{c}$ is the quadratic Casimir of the adjoint representation. Without adjoint matter, the constant $c_{1}$ is determined numerically to be $\approx 0.0281(3)$ in the continuum limit for $N_{c}=2$ 32], and the inclusion of adjoint matter leaves the value practically unchanged [33].

Infinitely thick brane: the broken symmetry phase. In the opposite limit of a $3 \mathrm{~d}$ broken symmetry phase, the three isospin components of the vector bosons convert into two massive vector bosons, of mass $m_{W}=g\left\langle\chi^{3}\right\rangle$, and one massless vector boson, the photon. In $3 \mathrm{~d}$, however, the photon becomes massive [34] via interactions with monopoles [35, 36],

$$
m_{\gamma} \sim g^{-3 / 2} m_{W}^{7 / 4} \exp \left(-\frac{2 \pi m_{W}}{g^{2}}\right) .
$$

Correspondingly, the potential is computable in weak coupling only up to distances $\sim m_{\gamma}^{-1}$. Assuming again, for simplicity, that $L_{z} \rightarrow \infty$, we obtain

$$
\begin{array}{rlrl}
F(R ; z) & =\frac{3}{4} \frac{g^{2}}{2 \pi R}, & & R \ll m_{W}^{-1}, \\
& =\frac{1}{4} \frac{g^{2}}{2 \pi R}, & & m_{W}^{-1} \ll R \ll m_{\gamma}^{-1}, \\
& =c_{2} g^{2} m_{\gamma}, \quad m_{\gamma}^{-1} \ll R \ll L_{r} / 2,
\end{array}
$$

where $c_{2}$ is a constant. If $L_{r} / 2 \ll m_{\gamma}^{-1}$, as is realistically the case, then at large distances we rather encounter the fully perturbative behaviour for the photon following from Eq. (4.2) (still in the limit $L_{z} \rightarrow \infty$ ),

$$
F(R ; z)=\frac{1}{4} \frac{g^{2}}{2 L_{r} \tan \left(\pi R / L_{r}\right)}, \quad m_{W}^{-1} \ll R .
$$


Brane of a finite width. Consider finally the case of our actual interest, a domain wall of some finite effective width, $\sim L_{\text {brane }}$. The behaviour should now interpolate between the two limits encountered above. The input parameter determining the width is $\alpha=\ell g^{2}$ as defined in Eq. (3.5). Independent of $\alpha$, at very small distances we still have

$$
F(R ; z)=\frac{3}{4} \frac{g^{2}}{2 \pi R}, \quad R \ll m_{W}^{-1} .
$$

What happens at large distances, on the other hand, depends on whether there is a zero-mode or not. If the zero-mode exists, we may expect that its contribution is according to Eq. (4.2),

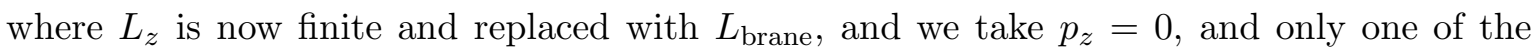
isospin components contributes so that $C_{F}=3 / 4 \rightarrow 1 / 4$,

$$
F^{(0)}\left(R ;|z| \lesssim L_{\text {brane }}\right) \sim \frac{g^{2}}{4} \frac{1}{2 L_{\text {brane }}}\left(1-\frac{2 R}{L_{r}}\right)
$$

This would be the behaviour if the confining phase outside the brane acted effectively as a Neumann (derivative of field vanishes), rather than Dirichlet (field itself vanishes), boundary condition. The first massive mode, on the other hand, contributes as

$$
\begin{aligned}
F^{(1)}\left(R ;|z| \lesssim L_{\text {brane }}\right) & \sim \frac{g^{2}}{4} \frac{1}{2 L_{\text {brane }}} \frac{\sinh \left[m_{1}\left(L_{r} / 2-R\right)\right]}{\sinh \left(m_{1} L_{r} / 2\right)} \\
& \approx \frac{g^{2}}{4} \frac{\exp \left(-m_{1} R\right)}{2 L_{\text {brane }}}, \quad R \ll L_{r} / 2, \quad m_{1} L_{r} \gg 1,
\end{aligned}
$$

where the mass $m_{1}$ is assumed non-zero. This would also be the full behaviour with Dirichlet boundary conditions, in which case a zero-mode is excluded.

Let us emphasize that the linear in $R$ behaviour of the force in Eq. (4.11) is a characteristic of the Abelian theory. If the $2 \mathrm{~d}$ low-energy effective theory were non-Abelian, the longdistance force would still be constant as in Eq. (4.4), with exponentially small finite-volume corrections. This difference is caused by the finite periodic extent of the box in the $R$ direction - in infinite volume both the $2 \mathrm{~d}$ Abelian and non-Abelian gauge theories display linear confinement (constant force) à la Eq. (4.4).

\section{Lattice formulation}

\subsection{Discretised action}

In order to test the behaviour at large distances, so as for instance to distinguish between Eqs. (4.11) and (4.13), we study the system on the lattice. The discretised Lagrangian corresponding to Eq. (3.2) is

$$
\mathcal{L}_{\text {latt }}=\frac{1}{a^{4} g^{2}} \sum_{k, l} \operatorname{Tr}\left[\mathbf{1}-P_{k l}(x)\right]
$$




$$
\begin{aligned}
& +\frac{2}{a^{2}} \sum_{k}\left[\operatorname{Tr} \chi^{2}(x)-\operatorname{Tr} \chi(x) U_{k}(x) \chi\left(x+a \hat{e}_{k}\right) U_{k}^{\dagger}(x)\right] \\
& +m_{\text {bare }}^{2}(z) \operatorname{Tr}\left[\chi^{2}\right]+\lambda\left(\operatorname{Tr}\left[\chi^{2}\right]\right)^{2},
\end{aligned}
$$

where $a$ is the lattice spacing, $U_{k}(x)=\exp \left[\operatorname{iag} A_{k}(x)\right], \hat{e}_{k}$ is a unit vector in direction $k$, and $P_{k l}$ is the plaquette:

$$
P_{k l}(x)=U_{k}(x) U_{l}\left(x+a \hat{e}_{k}\right) U_{k}^{\dagger}\left(x+a \hat{e}_{l}\right) U_{l}^{\dagger}(x) .
$$

The extents of the box are denoted by $L_{r}=a N_{r}, L_{t}=a N_{t}, L_{z}=a N_{z}$, and the lattice volume by $V=N_{r} N_{t} N_{z}$. The lattice spacing is expressed through the dimensionless combination

$$
\beta \equiv \frac{2 N_{c}}{a g^{2}}
$$

In order for physics to be the same as with the continuum Lagrangian in Eq. (3.2), the lattice theory needs to be renormalised. In three dimensions, the only parameter including divergences is $m^{2}$, and the divergences can be computed exactly, close to the continuum limit [37, 38]:

$$
\begin{aligned}
m_{\text {bare }}^{2}(z) \equiv & m^{2}(z)+\delta m^{2}(\bar{\mu}) \\
\delta m^{2}(\bar{\mu})= & -\left[2 g^{2} C_{A}+\lambda\left(d_{A}+2\right)\right] \frac{\Sigma}{4 \pi a} \\
& +\frac{1}{16 \pi^{2}}\left[2 \lambda\left(d_{A}+2\right)\left(\lambda-g^{2} C_{A}\right)\left(\ln \frac{6}{a \bar{\mu}}+\zeta\right)-2 g^{2} C_{A} \lambda\left(d_{A}+2\right)\left(\frac{\Sigma^{2}}{4}-\delta\right)\right. \\
& \left.-g^{4} C_{A}^{2}\left(\frac{5}{8} \Sigma^{2}+\left(\frac{1}{2}-\frac{4}{3 C_{A}^{2}}\right) \pi \Sigma-4(\delta+\rho)+2 \kappa_{1}-\kappa_{4}\right)\right]
\end{aligned}
$$

where the constants $\zeta, \delta, \rho, \kappa_{1}, \kappa_{4}$ and $\Sigma$ have been defined in Refs. 37, 38, $C_{A}=N_{c}$, $d_{A}=N_{c}^{2}-1$, and $\bar{\mu}$ is the scale parameter of the $\overline{\mathrm{MS}}$ scheme. The couplings $g^{2}, \lambda$ require no renormalisation, but the approach to the continuum limit could be improved by computing corrections of order $\mathcal{O}(a)$ 39]. As already mentioned, we choose $\bar{\mu}=g^{2}$ in the following. Various condensates, such as $\operatorname{Tr}\left[\chi^{2}\right]$, also require additive renormalisation (multiplicative wave function renormalisation effects are $\mathcal{O}(a)$ [39]):

$$
\left\langle\operatorname{Tr}\left[\chi^{2}\right]\right\rangle_{\overline{\mathrm{MS}}}=\left\langle\operatorname{Tr}\left[\chi^{2}\right]\right\rangle_{\mathrm{bare}}-d_{A} \frac{\Sigma}{8 \pi a}-d_{A} C_{A} \frac{g^{2}}{16 \pi^{2}}\left(\ln \frac{6}{a \bar{\mu}}+\zeta+\frac{\Sigma^{2}}{4}-\delta\right) .
$$

\subsection{Discretised observables}

The basic object we employ on the lattice is a Polyakov loop,

$$
P(R ; z) \equiv \operatorname{Tr}\left[\Pi_{n_{t}=0}^{N_{t}-1} U_{t}\left(R, n_{t} a, z\right)\right],
$$


and the correlation function of two Polyakov loops is

$$
C(R ; z) \equiv \sum_{n_{r}=0}^{N_{r}-1}\left\langle P^{\dagger}\left(n_{r} a ; z\right) P\left(n_{r} a+R ; z\right)\right\rangle .
$$

The general structure of this correlator is (31] and references therein)

$$
C(R ; z)=w_{1} e^{-L_{t} V(R ; z)}+w_{2} e^{-L_{t}[V(R ; z)+\Delta E]}+\ldots,
$$

where $w_{i}$ are numerical coefficients and $\Delta E$ is a mass gap. Therefore the static force, to be denoted by $F_{P}(R ; z)$ when extracted from the Polyakov loop correlator, can be defined as

$$
F_{P}(R ; z) \equiv \frac{1}{L_{t}} \frac{\mathrm{d}}{\mathrm{d} R} \ln C(R ; z),
$$

and the difference between $F_{P}(R ; z)$ and $F(R ; z)$ is exponentially small if $\Delta E>0$ and $L_{t}$ is large enough. On the lattice we use a discretised version of Eq. (5.10),

$$
F_{P}\left(R+\frac{a}{2} ; z\right) \equiv \frac{2}{a L_{t}} \frac{C(R+a ; z)-C(R ; z)}{C(R+a ; z)+C(R ; z)} .
$$

The reason for using Polyakov loop correlators instead of the more common Wilson loop to obtain the static force, is simply that a sufficient numerical accuracy is easier to reach, thanks to the advanced numerical techniques developed by Lüscher and Weisz [31] (and optimised for the present system by us). The measurement method will be described below. This advantage does not come without a price, however: if the system is not confining, then the error made by using Eq. (5.10) rather than the full expression in Eq. (5.9) may in general not be small, and the correct result is only obtained after an extrapolation to the limit of a large $L_{t}$. Nevertheless, even data obtained at finite $L_{t}$ will correctly show whether the behaviour of the force is constant, ${ }^{6}$ linear, or exponential in $R$, thus allowing to distinguish between Eqs. (4.4), (4.11), (4.13).

In the following, we will always refer to the object defined by Eqs. (5.10), (5.11) as the static force, while keeping the reservations just spelled out in mind, and therefore checking in the end explicitly for the stability of our results with respect to variations of $L_{t}$.

\subsection{Parameters and systematics}

The model of Eq. (5.1) has previously been studied with lattice simulations in Refs. 41, 42, for the case of a homogeneous mass parameter, corresponding to $\alpha=0$ or $\alpha=\infty$. The system was observed to have a non-trivial phase diagram in the space of the continuum parameters

\footnotetext{
${ }^{6}$ At large distances the behaviour of $F_{P}$ deviates from a constant, due to the periodic boundary conditions. Based on the effective string picture, the behaviour expected is the same as in $2 \mathrm{~d}\left[40, F_{P}(R ; z)=\right.$ $\sigma \tanh \left[\sigma L_{t}\left(\frac{L_{r}}{2}-R\right)\right]$ for large $L_{r}$, which approaches a step function for $L_{t} \rightarrow \infty$. In practice, however, the error bars are reasonably small only for $R \ll L_{r} / 2$, where $F_{P}$ can be well approximated with a constant.
} 
$x, y$, defined in Eq. (3.5): for $x<x_{c} \approx 0.3$, there is a first order phase transition separating the broken symmetry phase at $y<y_{c}$ from the symmetric phase at $y>y_{c}$. For $x>x_{c}$, on the other hand, there is no phase transition, meaning that the two phases are analytically connected. This is possible since there are monopoles in the broken symmetry phase [35, 36] which replace a possible order parameter, the mass of a photon related to an unbroken $\mathrm{U}(1)$ symmetry, by a small but non-vanishing mass (given in Eq. (4.5)) related to a pseudoscalar particle [34. The situation would be different with the gauge group SU(3), for instance, in which case there is an additional discrete global symmetry $\chi \rightarrow-\chi$ which gets broken at $y=y_{c}$; thus there always is a genuine phase transition of some kind [43].

In terms of the parameters of Eq. (3.5), we choose here to study the value $x=0.20$, which implies a first order transition at $y_{c} \approx 0.147$ [42] (provided one is close to the continuum limit, i.e., $\beta \gg 1$ ). The motivation is to have a clear distinction between the two phases, and to avoid complications owing to the presence of monopoles, which are more important in the crossover region. The parameters $y_{1}, y_{2}$ of Eq. (3.5) are chosen on the two sides of the transition,

$$
y_{1}=-0.667, \quad y_{2}=2.0 .
$$

The width of the domain wall is varied in small intervals in the range $\alpha=\ell g^{2}=0.0 \ldots 5.3$, and we also have data for $\alpha=\infty$.

In order to control finite lattice spacing and finite volume artifacts, we have carried out simulations at two values of $\beta, \beta=6,9$, and with a series of volumes in the range $V=$ $N_{r} N_{t} N_{z}=24^{3} \ldots 48^{3}$. Experiences from previous studies of the same system [41, 42, 33] as well as from glueball computations with pure $\mathrm{SU}(2)$ gauge theory in three dimensions [32] suggest that such values are already safely in the scaling region.

The update algorithm used for the simulations is a combination of one heat bath update cycle followed by four overrelaxation updates for both the gauge and the adjoint scalar fields. More details concerning the implementation can be found in Ref. [42].

\subsection{Measuring the Polyakov loop correlation functions}

In a confining system at large $L_{t}$ the Polyakov loop correlation function is a very "noisy" observable: the magnitude of the correlation function is $\sim \exp \left(-\sigma L_{t} R\right)$, whereas the noise is always of order unity. Thus, without any advanced techniques huge statistics is needed. We employ here a modification of the multilevel approach presented in Ref. 31].

To summarize our method, let us again consider a lattice of size $N_{r} \times N_{t} \times N_{z}$, where the Polyakov loops are oriented along the $t$-direction and the correlation function is to be measured in the $r$-direction. One measurement cycle works as follows:

1. Divide the lattice into $N_{t}$ sublattices by freezing the following variables:

(a) gauge link variables $U_{r}$ and $U_{z}$ and adjoint scalars $\chi$ which reside on the $(r, z)$-planes 
located at $t / a=0,2, \ldots N_{t}-2,{ }^{7}$ and

(b) $U_{t}, U_{z}$ and $\chi$ on $(t, z)$-planes at $r=0$ and $r / a=N_{r} / 2$.

2. Perform $N$ update sweeps on the non-frozen variables within all the sublattices. Because the boundaries of the sublattices are fixed, we can generate a valid full lattice configuration by choosing any of the $N$ configurations from each sublattice, giving a total of $N^{N_{t}}$ lattice configurations.

3. The Polyakov loop correlation function is measured so that one of the loops is located in the interval $0<r_{1} / a<N_{r} / 2$ and the other at $N_{r} / 2<r_{2} / a<N_{r}$. This gives effectively $N^{N_{t}}$ measurements; the exponential growth with $N_{t}$ compensates for the exponential decrease in the signal.

4. In practice, one improved correlation function measurement is (we suppress the $z$ coordinate)

$$
\bar{C}\left(r_{2}-r_{1}\right)=\bar{P}^{\dagger}\left(r_{1}\right) \bar{P}\left(r_{2}\right), \quad \bar{P}(r)=\operatorname{Tr}\left[\bar{S}(0, r) \bar{S}(2 a, r) \ldots \bar{S}\left(\left(N_{t}-2\right) a, r\right)\right],
$$

where $\bar{S}$ is the average of the 2-link piece of the Polyakov loop within one sublattice:

$$
\bar{S}(t, r)=\frac{1}{N} \sum_{i=1}^{N} U_{t}^{(i)}(t, r) U_{t}^{(i)}(t+a, r) .
$$

Clearly, $\bar{S}$ is an average over $N, \bar{P}$ over $N^{N_{t} / 2}$ and $\bar{C}\left(r_{2}-r_{1}\right)$ over $N^{N_{t}}$ measurements. The results are further averaged over $r_{1}$ while $R=r_{2}-r_{1}$ is kept fixed. However, $r_{1}$ and $r_{2}$ should not be too close to the fixed $r=0$ and $r / a=N_{r} / 2$ planes, which would diminish the configuration-by-configuration variation and hence the noise reduction. Thus, the technique works best when $R / a$ is largest, $\sim N_{r} / 2$, just where it is needed.

The parameter $N$ is to be optimized for the physical situation in question; the range we used is $N \sim 100-300$. Naturally, the $N$ configurations are far from independent; thus, standard update cycles must be performed too. Nevertheless, the method achieves the goal of strongly reducing the statistical noise. Clearly, maximizing the number of sublattices achieves the highest averaging. However, $t$-slices of thickness 1 (which would yield $N^{2 N_{t}}$ measurements) are not very useful, because the variables within blocks would be too strongly locked in place.

The difference between the method above and the one presented in Ref. 31] is that the latter does not implement the freezing of the planes in step 1(b) above. The number of measurements is thus cut to $N^{N_{t} / 2}$; on the other hand a larger optimal $N$ tends to compensate for this. In our case the existence of the scalar field $\chi$ in general lowers the useful values for $N$ - indeed, in the broken phase the whole improvement becomes largely unnecessary.

Let us end this section with another technical comment. Before starting the measurements, the system needs to be "thermalised". That is, one starts from some initial configuration

\footnotetext{
${ }^{7}$ We assume that indices start from zero, i.e., $t / a=0 \ldots N_{t}-1$, for example.
} 


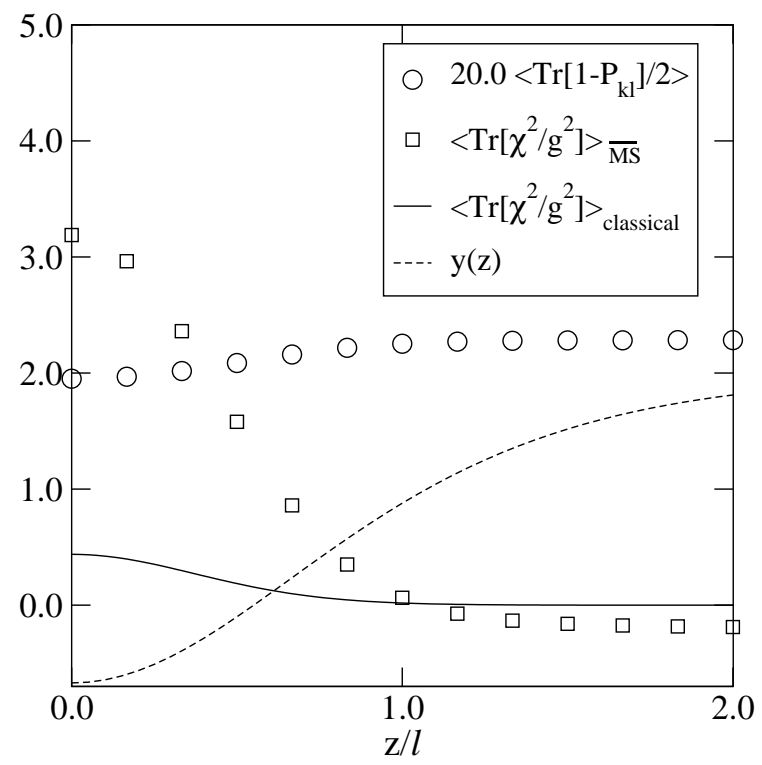

Figure 1: Various condensates, as well as the mass parameter $y(z)$, as a function of $z / \ell$ at $\alpha=\ell g^{2}=2.667, \beta=9, V=48^{2} \times 32$. In a homogeneous system, the phase transition point is at $y(z)=y_{c} \approx 0.15$ [2]. The classical prediction for the scalar condensate, obtained by solving the equations of motion numerically, is also shown.

(for instance "cold", whereby all fields are frozen to values corresponding to the classical minimum of the action, or "hot", whereby all fields have random values) and carries out updates until the system has reached a typical "fluctuating" configuration, which should be independent of the initial one. It now turns out that with a "hot" start, the thermalisation process is anomalously slow. The reason is that the system can contain $\mathrm{U}(1)$ vortices and anti-vortices penetrating through the domain wall. Since there is no net magnetic flux, all the vortices and anti-vortices must eventually evaporate, but microscopic updates are very slow in achieving such a global change in practice. With a "cold" start, on the contrary, there is no problem with thermalisation, and it is thus the method of choice for this system.

\section{Results}

We now move on to discuss our numerical results. To start with, we show in Fig. 1 the actual structure of the domain wall for typical parameter values, in terms of local condensates. It is seen that, judging by the eye, the width of the domain wall is indeed well determined by the input parameter $\ell$, as defined through Eq. (3.3). There is quite a difference, though, in the magnitudes of the lattice value of the condensate (square boxes) and the tree-level value (continuous line). The difference can be explained by the fact that with our choice of parameters higher order corrections to condensates are of the same order as the tree-level 


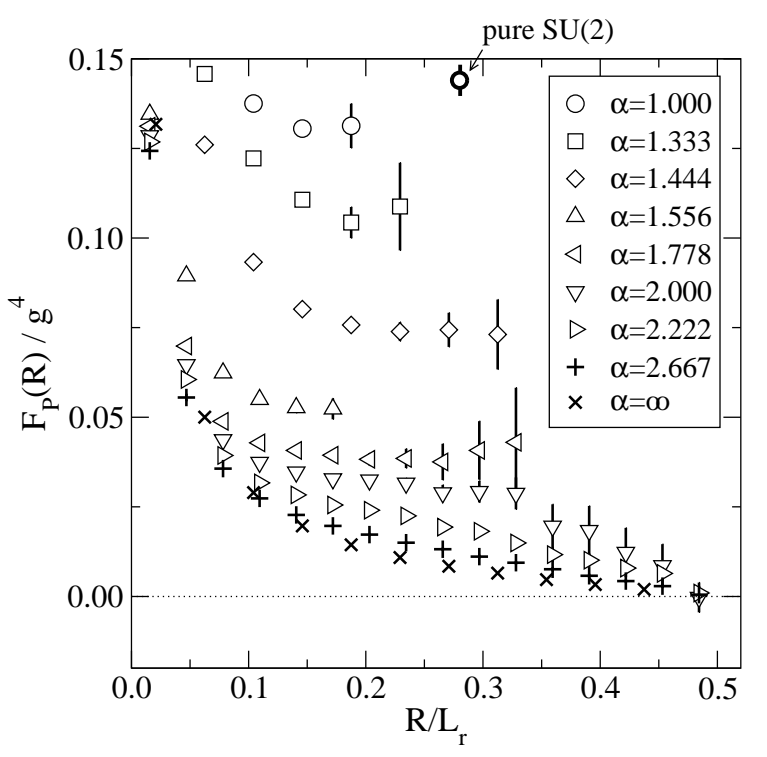

Figure 2: The static force for various values of $\alpha$, at $\beta=6$, as a function of $R / L_{r}$. When not visible, the error bars are smaller than the symbol sizes. The volume is $V=24^{3}$ for $\alpha=\infty$, and $V=32^{2} \times 24$ otherwise. The pure gauge value at $\beta=6$ is taken from Ref. 32.

value, so that perturbation theory cannot be trusted. We have checked (in the limit of a thick domain wall) that an account of 1-loop effects makes the difference considerably smaller.

Our basic observable, the force $F_{P}(R)$ in the central plane $z=0$, is shown in Fig. 2, for various values of $\alpha=\ell g^{2}$. For $\alpha=0$, corresponding to $3 \mathrm{~d}$ confinining behaviour, the force becomes constant (or rather tanh-like) at intermediate distances, representing the $3 \mathrm{~d}$ string tension. Measurements are relatively difficult, since the Polyakov loop correlation function decays rapidly with $R$. As $\alpha$ is increased, the interior of the domain wall starts to look more and more like a homogeneous broken symmetry phase, and the plateau moves down, eventually disappearing completely. The force then resembles the behaviour observed in the 3 d broken symmetry phase, Eq. (4.9), corresponding to $\alpha=\infty$.

The quantity we would like to extract from curves of the type in Fig. 2 will be referred to as the "string tension". There are, however, two qualitatively different regimes for the behaviour observed: for small $\alpha$, the domain wall is essentially in the symmetric phase and we extract the string tension from the plateau in $F_{P}(R)$ at $R \ll L_{r}$. On the other hand, for larger $\alpha$ the domain wall is in the broken symmetry phase, and the string tension is extracted from the coefficient of a "linear term" in $F_{P}(R)$, that is, from $F_{P}(R) /\left[1-2 R / L_{r}\right]$ at $R \approx L_{r} / 2$. It turns out that, as a remnant of the first order transition experienced by the homogeneous system at $x=0.20$, for certain values of $\alpha$ the central plane can even be in one of two metastable branches, exhibiting these two patterns. This situation is illustrated in Fig. 3(left). 

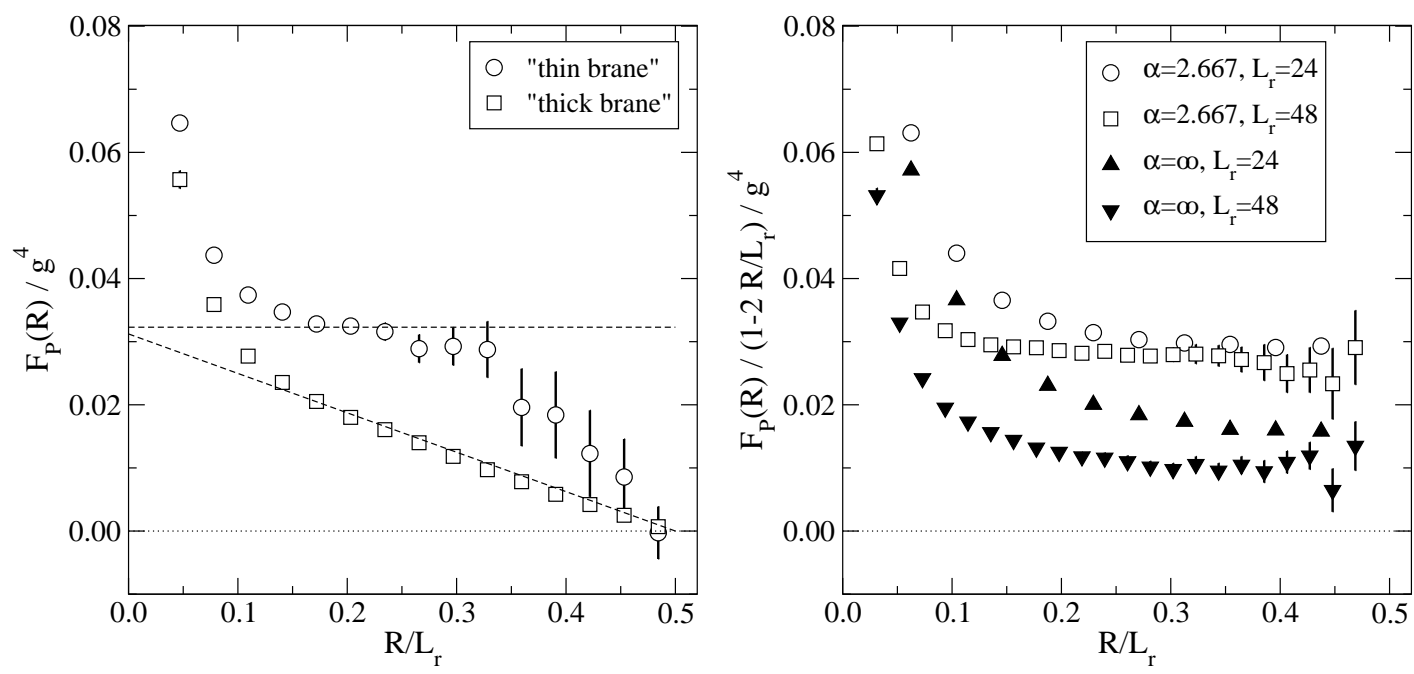

Figure 3: Left: The static force in the central plane in two different runs, at $\ell g^{2}=2, \beta=6$, $V=32^{2} \times 24$. The system can be in one of two metastable states, resulting in different qualitative behaviours for the force. These are referred to as the "thin brane" and the "thick brane", because the former has the functional form appearing in the limit $\ell \rightarrow 0$, the latter in the limit $\ell \rightarrow \infty$. Right: The "thick brane" static force, divided by $\left(1-2 R / L_{r}\right)$, at different values of $L_{r}$, compared with results for the homogeneous broken symmetry phase. The parameters are $\beta=6, V=L_{r} \times 24^{2}$.

While a force constant in $R$ at intermediate distances is a signal of non-Abelian $3 \mathrm{~d}$ confinement, a behaviour linear in $1-2 R / L_{r}$ as $R \rightarrow L_{r} / 2$ still allows for two different interpretations: 3d Coulomb phase, characterised by Eq. (4.9), and $2 \mathrm{~d}$ Coulomb phase, characterised by Eq. (4.11). One can differentiate between the two by approaching $R \approx L_{r} / 2$, where

$$
\begin{aligned}
\frac{\sigma}{g^{4}} \equiv \lim _{R \rightarrow \frac{L_{r}}{2}} \frac{1}{g^{4}}\left[\frac{F_{P}(R)}{1-2 R / L_{r}}\right] & =\frac{3 \pi}{16 g^{2} L_{r}}, \quad 3 \mathrm{~d} \mathrm{U}(1), \\
& =\frac{1}{8 g^{2} L_{\text {brane }}}, \quad 2 \mathrm{~d} \mathrm{U}(1) .
\end{aligned}
$$

Therefore, if we increase the system size $L_{r}$, the existence of a $2 \mathrm{~d}$ zero-mode is signalled by the force approaching a constant, ${ }^{8}$ while for a $3 \mathrm{~d}$ Coulomb phase the apparent "string tension" vanishes in the infinite volume limit as $\sim 1 / L_{r}$, and the potential becomes a power law, Eq. (4.7). These different qualitative behaviours are illustrated in Fig. 3(right). Thus, whether dimensional reduction takes place or not can be seen by monitoring the dependence of the observable in Eq. (6.1) on the box size $L_{r}$.

Results for the string tension, extracted either from a plateau in the static force $F_{P}(R)$, or as defined in Eq. (6.1), are shown as a function of $\alpha$ in Fig. (4. We observe that the behaviour

\footnotetext{
${ }^{8}$ Let us stress again that the existence of a string tension in an Abelian theory is specific to $2 \mathrm{~d}$.
} 


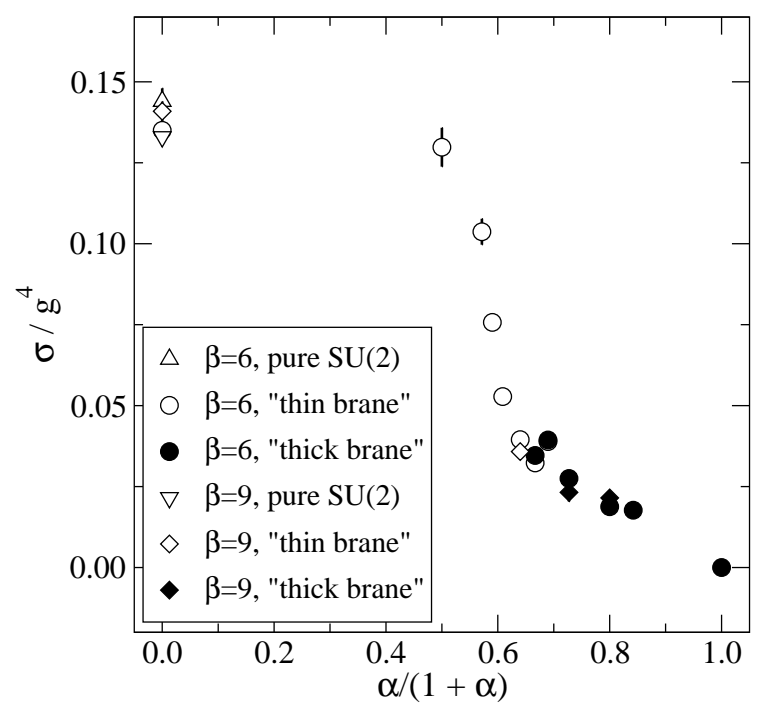

Figure 4: The string tension, $\sigma / g^{4}$, as a function of $\alpha /(1+\alpha)$. Pure gauge values are from Ref. 32]. The physical volume for the three smallest $\alpha$ 's is $g^{6} a^{3} V=16^{2} \times 10.67$, otherwise $g^{6} a^{3} V=21.33^{2} \times 16$. The labels "thin brane" and "thick brane" refer to whether the functional form of the data points resembles the upper or lower curve in Fig. 3(left), which in turn determines the fit ansatz employed (see the text).

interpolates between the $3 \mathrm{~d}$ confinement and $3 \mathrm{~d}$ Coulomb behaviours. For narrow domain walls, or small $\alpha \lesssim 2.0$, the system behaves as a $3 \mathrm{~d}$ confinement phase ("thin brane"). For larger $\alpha$, it is dominated by a $2 \mathrm{~d}$ zero-mode ("thick brane"), with a string tension scaling as $\sim 1 / L_{\text {brane, }}$ as in Eq. (6.2). Note that the system goes over into the "thick brane" regime only when the string tension is a factor 3...4 smaller than in the bulk. Increasing $\alpha$ further, the string tension continues to decrease, but the system starts simultaneously to look more and more like a $3 \mathrm{~d}$ Coulomb phase, to which it finally goes over as $\alpha \rightarrow \infty$.

As discussed above, to make a rigorous distinction between a $2 \mathrm{~d}$ Coulomb and $3 \mathrm{~d}$ Coulomb behaviour, one has to carry out a finite-size scaling study, as in Fig. 3(right). The corresponding $\sigma$ 's are shown in the top part of Table 1 it is again seen clearly that at $\alpha=2.667$ the string tension is indeed independent of $L_{r}$, while at $\alpha=\infty$ it is not.

Table 1 also serves to demonstrate that our results are independent of the extents of the system in all the directions, for a given lattice spacing. The independence of the lattice spacing, for a given physical volume, is demonstrated by Fig. 4

The discussion so far has been for the force in the central plane, $z=0$. In Fig. 5 we show how the force depends on $z$. The pattern is as expected: the behaviour characteristic of the properties of the $2 \mathrm{~d}$ zero-mode is well localised, and outside of the domain wall the dynamics is that of the bulk theory.

All the evidence presented so far supports the conjecture that the low-energy dynamics on 


\begin{tabular}{cccc}
\hline$\alpha=2.667$ & \multicolumn{3}{c}{$\alpha=\infty$} \\
\hline$V=L_{r} \times 24 \times 24$ & $\sigma / g^{4}$ & $V=L_{r} \times 24 \times 24$ & $\sigma / g^{4}$ \\
\hline$L_{r}=24$ & $0.029(1)$ & $L_{r}=24$ & $0.0160(5)$ \\
$L_{r}=36$ & $0.031(2)$ & $L_{r}=36$ & $0.0110(7)$ \\
$L_{r}=48$ & $0.028(1)$ & $L_{r}=48$ & $0.0098(5)$ \\
\hline$V=32 \times L_{t} \times 16$ & $\sigma / g^{4}$ & & \\
\hline$L_{t}=16$ & $0.028(2)$ & & \\
$L_{t}=24$ & $0.026(2)$ & & \\
$L_{t}=32$ & $0.030(3)$ & & \\
$L_{t}=40$ & $0.028(2)$ & & \\
\hline$V=32 \times 32 \times L_{z}$ & $\sigma / g^{4}$ & & \\
\hline$L_{z}=16$ & $0.030(3)$ & & \\
$L_{z}=24$ & $0.028(2)$ & & \\
$L_{z}=32$ & $0.029(1)$ & & \\
\hline
\end{tabular}

Table 1: Finite-volume scaling of $\sigma / g^{4}$ as defined in Eq. (6.1), at $\beta=6, \alpha=2.667$ (left), and $\alpha=\infty$ (right).

the brane is determined by a localised $2 \mathrm{~d}$ zero-mode. In order to understand the distance scale at which corrections to the zero-mode dynamics become important, however, one should also determine the mass gap to the higher modes in the central plane. To achieve this, we define the quantity

$$
m_{\mathrm{eff}}(R) \equiv-\frac{F_{P}^{\prime \prime}(R)}{\left[F_{P}(R) /(1-2 R / L)\right]^{\prime}},
$$

where $[\ldots]^{\prime} \equiv \mathrm{d}[\ldots] / \mathrm{d} R$. According to Eqs. (4.11), (4.13), the zero-mode does not contribute to this observable, and the leading non-trivial contribution at large distances is $m_{\text {eff }}(R) \approx m_{1}$.

In Fig. 6 we plot a discretised version of $m_{\mathrm{eff}}(R)$, in the dimensionless form $\ell m_{\mathrm{eff}}(R)$. Thus, if $m_{1} \sim 1 / L_{\text {brane }} \propto 1 / \ell$, we should obtain a constant value, independent of $\ell$. This indeed is the behaviour observed, within statistical errors. Moreover, the distance where the plateau value is reached is $R / \ell \sim 1$, consistent with the scenario that the masses of higher modes still also scale as $1 / \ell$, as expected for "Kaluza-Klein type" excitations.

\section{Conclusions and outlook}

The purpose of this paper has been to test with numerical lattice Monte Carlo simulations some basic features of a mechanism proposed for gauge field localisation on domain wall defects by Dvali and Shifman [26]. The mechanism relies on non-perturbative dynamics in 

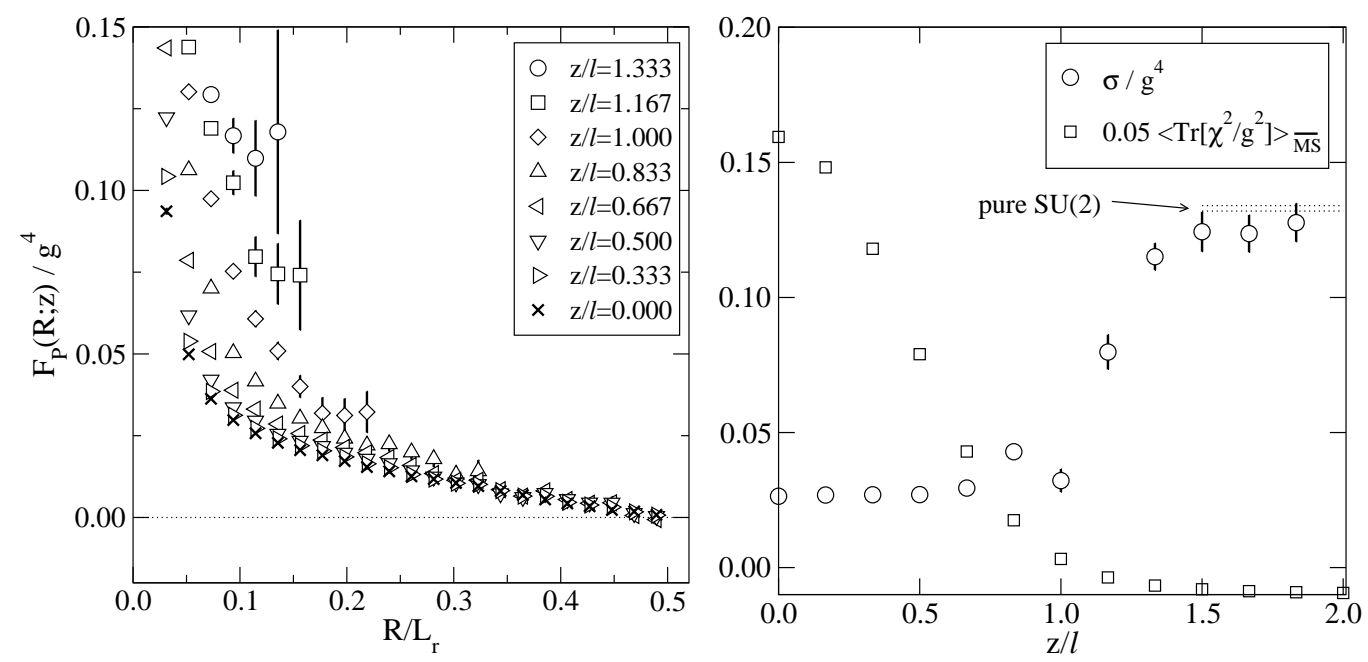

Figure 5: Left: The static force in various planes, for $\ell g^{2}=2.667, \beta=9, V=48^{2} \times 32$. Right: The corresponding string tensions, $\sigma / g^{4}$, together with the scalar condensate. For $z / \ell \geq 1.0(z / \ell<1.0)$, the fit is of the "thin brane" ("thick brane") type in Fig. 3(left).

the "bulk" outside of the domain wall, and is thus not easily "proven" to work with analytic methods alone.

Dvali and Shifman considered originally a $3+1$ dimensional $\mathrm{SU}(2)$ gauge theory, arguing that the low-energy dynamics was that of $2+1$ dimensional $\mathrm{U}(1)$ gauge theory. For technical reasons, and since the main physics arguments remain essentially unchanged, we simplified in this paper the setting further, and took as the starting point a $2+1$ dimensional $\mathrm{SU}(2)$ gauge theory, with adjoint scalar matter.

The basic pattern we found can be summarised as follows (cf. Fig. 4). Suppose the $2+1$ dimensional theory has a large confinement scale $\Lambda$, and consider the dynamics on the brane. If the thickness of the brane is of order unity with respect to $\Lambda^{-1}$, the dynamics remains the same as in $2+1$ dimensions, but the effective string tension decreases rather rapidly with the thickness (open symbols in Fig. 4). As the brane is made thicker, the dynamics becomes finally $1+1$ dimensional (closed symbols in Fig. 4), with the "confinement scale" (or string tension, $\sqrt{\sigma}$ ) taking over (almost) smoothly from the $2+1$ dimensional value. However the transition between the two behaviours actually seems to be discontinuous in our system (cf. Fig. 3(left)). Increasing the width further, $\sqrt{\sigma}$ goes down as $\sim 1 / \sqrt{L_{\text {brane }}}$, while the mass

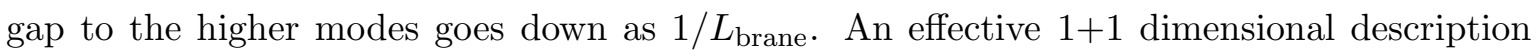
with an extremely small $\sqrt{\sigma} \ll \Lambda$ is only reached for $L_{\text {brane }} \gg \Lambda^{-1}$, and is then valid only at distances $\gg L_{\text {brane }}$ which implies a kind of a hierarchy problem: there have to be two different large scales compared with the dynamical energy scales of the zero-mode system (such as masses of bound states, or $\sqrt{\sigma}$ ), namely $L_{\text {brane }}^{-1}$ and $\Lambda$. Moreover, scales such as $\sqrt{\sigma}$ are larger than the masses of some of the higher modes, which means that the contributions 


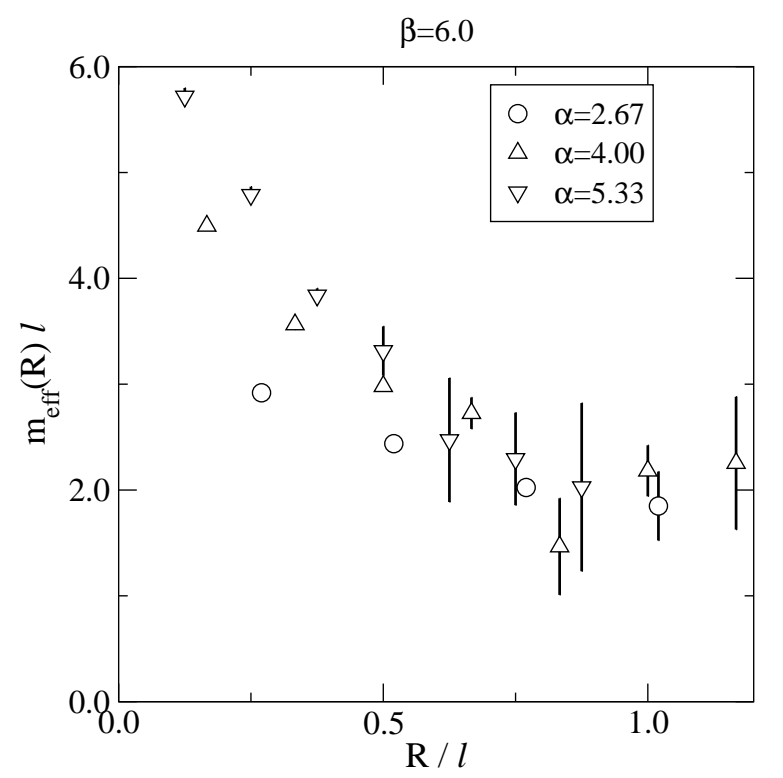

Figure 6: The observable $m_{\mathrm{eff}}(R)$ defined in Eq. (6.3), multiplied by $\ell$, as a function of $R / \ell$, for various $\ell\left(\alpha=\ell g^{2}\right)$. The independence of $\alpha$ at large $R \gtrsim \ell$ demonstrates that $m_{\mathrm{eff}} \sim 1 / \ell$.

from the latter are not suppressed in generic infrared observables.

How would this pattern change in $3+1$ dimensions? If we consider the very scenario studied by Dvali and Shifman, then there is in fact a clear difference. The reason is that the $2+1$ dimensional $\mathrm{U}(1)$ theory no longer has a string tension associated with a linearly rising potential (ignoring the exponentially small value produced by the Polyakov mechanism). Thus, repeating our measurements, one would indeed expect to observe a qualitative change in the low-energy dynamics, once the width of the domain wall is somewhat wider than the inverse of the confinement scale outside the brane: the closed symbols in Fig. 4 would all lie on an (almost) straight line, at (almost) vanishing $\sigma$, signalling a phase transition between the bulk-like "thin brane" and localised "thick brane" regimes.

On the other hand, if we rather consider a non-Abelian case, say a bulk SU(3) theory, such that the low-energy dynamics is that of $2+1$ dimensional $\mathrm{SU}(2) \times \mathrm{U}(1)$ theory, and probe the properties of the non-Abelian part, then the pattern should again be largely similar to what we found in this paper. In other words, the domain wall has to be wider than the inverse of the bulk confinement scale for a $2+1$ dimensional zero-mode to exist, but in general the confinement scale of the corresponding low-energy effective theory is smaller than that in the bulk only by some numerical factor roughly of order unity, unless the domain wall is significantly wider than the inverse of the bulk confinement scale. In the latter case the masses of localised bound states (or, say, the string tension $\sqrt{\sigma}$ ) are expected to scale as $\sim g^{2} \sim g_{4}^{2}\left(\ell^{-1}\right) \ell^{-1}$, where $g_{4}^{2}(\bar{\mu})$ is the renormalised $4 \mathrm{~d}$ gauge coupling. For $1 / \ell$ much below the $4 \mathrm{~d}$ Yang-Mills confinement scale they are thus of the same order of magnitude or larger 
than the lightest of the higher modes, with masses $\sim 1 / \ell$.

The situation in the $3+1$ dimensional case can perhaps be illustrated by noting that, in a way, the present mechanism is analogous to the familiar dimensional reduction of $4 \mathrm{~d}$ YangMills theory at a finite temperature $T$, which occurs only for $T$ above the deconfinement phase transition temperature. The role of $T$ is played by $\ell^{-1}$. The conflict arises because we now need a domain wall wider than the bulk confinement scale, corresponding to $T$ below the deconfinement phase transition, thus no dimensional reduction.

It would naturally be very interesting to understand whether the patterns observed in this paper change if we go to the physically interesting $4+n$ dimensional case, with $n \geq 1$.

\section{Acknowledgements}

We thank D. Bödeker, P. de Forcrand and M. Shifman for helpful discussions. This work was partly supported by the RTN network Supersymmetry and the Early Universe, EU Contract No. HPRN-CT-2000-00152, by the Swiss Science Foundation, and by the Academy of Finland, grant No. 104382. H.M. thanks the University of Lausanne for the generous Bourse de perfectionnement et de recherche.

\section{References}

[1] V.A. Rubakov and M.E. Shaposhnikov, Phys. Lett. B 125 (1983) 136.

[2] K. Akama, Lect. Notes Phys. 176 (1982) 267 hep-th/0001113.

[3] L. Randall and R. Sundrum, Phys. Rev. Lett. 83 (1999) 4690 hep-th/9906064.

[4] N. Arkani-Hamed, S. Dimopoulos and G.R. Dvali, Phys. Lett. B 429 (1998) 263 hep-ph/9803315; I. Antoniadis, N. Arkani-Hamed, S. Dimopoulos and G.R. Dvali, Phys. Lett. B 436 (1998) 257 hep-ph/9804398; N. Arkani-Hamed, S. Dimopoulos and G.R. Dvali, Phys. Rev. D 59 (1999) 086004 hep-ph/9807344.

[5] O. DeWolfe, D.Z. Freedman, S.S. Gubser and A. Karch, Phys. Rev. D 62 (2000) 046008 hep-th/9909134.

[6] M. Giovannini, H. Meyer and M.E. Shaposhnikov, Nucl. Phys. B 619 (2001) 615 hep-th/0104118.

[7] E. Roessl and M. Shaposhnikov, Phys. Rev. D 66 (2002) 084008 hep-th/0205320.

[8] S. Randjbar-Daemi and M. Shaposhnikov, Nucl. Phys. B $645 \quad$ (2002) 188 hep-th/0206016. 
[9] V.A. Rubakov, Phys. Usp. 44 (2001) 871 [Usp. Fiz. Nauk 171 (2001) 913] hep-ph/0104152.

[10] V.A. Rubakov and M.E. Shaposhnikov, Phys. Lett. B 125 (1983) 139.

[11] B. Bajc and G. Gabadadze, Phys. Lett. B 474 (2000) 282 hep-th/9912232.

[12] I. Oda, Phys. Lett. B 496 (2000) 113 hep-th/0006203.

[13] S. Randjbar-Daemi and M.E. Shaposhnikov, Phys. Lett. B 492 (2000) 361 hep-th/0008079.

[14] A. Kehagias and K. Tamvakis, Phys. Lett. B 504 (2001) 38 hep-th/0010112.

[15] A. Neronov, Phys. Rev. D 65 (2002) 044004 gr-qc/0106092.

[16] S. Randjbar-Daemi and M. Shaposhnikov, JHEP 04 (2003) 016 hep-th/0303247.

[17] S.L. Dubovsky, V.A. Rubakov and P.G. Tinyakov, JHEP 08 (2000) 041 hep-ph/0007179.

[18] A. Neronov, Phys. Rev. D 64 (2001) 044018 hep-th/0102210.

[19] M. Giovannini, Phys. Rev. D 66 (2002) 044016 hep-th/0205139.

[20] D.B. Kaplan, Phys. Lett. B 288 (1992) 342 hep-lat/9206013.

[21] S.L. Dubovsky and V.A. Rubakov, Int. J. Mod. Phys. A 16 (2001) 4331 hep-th/0105243.

[22] M.E. Shaposhnikov and P. Tinyakov, Phys. Lett. B 515 (2001) 442 hep-th/0102161.

[23] M. Laine, H.B. Meyer, K. Rummukainen and M. Shaposhnikov, JHEP 01 (2003) 068 hep-ph/0211149.

[24] P. Dimopoulos, K. Farakos, A. Kehagias and G. Koutsoumbas, Nucl. Phys. B 617 (2001) 237 hep-th/0007079.

[25] M. Shifman and A. Yung, hep-th/0312257.

[26] G.R. Dvali and M.A. Shifman, Phys. Lett. B 396 (1997) 64 and B 407 (1997) 452 (E) hep-th/9612128.

[27] M. Creutz, Phys. Rev. Lett. 43 (1979) 553 and 43 (1979) 890 (E).

[28] K. Farakos, P. de Forcrand, C.P. Korthals Altes, M. Laine and M. Vettorazzo, Nucl. Phys. B 655 (2003) 170 hep-ph/0207343; H. Gies, Phys. Rev. D 68 (2003) 085015 hep-th/0305208. 
[29] N. Arkani-Hamed and M. Schmaltz, Phys. Lett. B 450 (1999) 92 hep-th/9812010.

[30] N. Tetradis, Phys. Lett. B 479 (2000) 265 hep-ph/9908209.

[31] M. Lüscher and P. Weisz, JHEP 09 (2001) 010 hep-lat/0108014 and 07 (2002) 049 hep-lat/0207003.

[32] M.J. Teper, Phys. Rev. D 59 (1999) 014512 hep-lat/9804008.

[33] A. Hart and O. Philipsen, Nucl. Phys. B 572 (2000) 243 hep-lat/9908041.

[34] A.M. Polyakov, Phys. Lett. B 59 (1975) 82; Nucl. Phys. B 120 (1977) 429.

[35] G. 't Hooft, Nucl. Phys. B 79 (1974) 276.

[36] A.M. Polyakov, JETP Lett. 20 (1974) 194 [Pisma Zh. Eksp. Teor. Fiz. 20 (1974) 430].

[37] K. Farakos, K. Kajantie, K. Rummukainen and M. Shaposhnikov, Nucl. Phys. B 442 (1995) 317 hep-lat/9412091.

[38] M. Laine, Nucl. Phys. B 451 (1995) 484 hep-lat/9504001; M. Laine and A. Rajantie, Nucl. Phys. B 513 (1998) 471 hep-lat/9705003.

[39] G.D. Moore, Nucl. Phys. B 493 (1997) 439 hep-lat/9610013 and B 523 (1998) 569 hep-lat/9709053.

[40] B.E. Rusakov, Mod. Phys. Lett. A 5 (1990) 693.

[41] A. Hart, O. Philipsen, J.D. Stack and M. Teper, Phys. Lett. B 396 (1997) 217 hep-lat/9612021.

[42] K. Kajantie, M. Laine, K. Rummukainen and M.E. Shaposhnikov, Nucl. Phys. B 503 (1997) 357 hep-ph/9704416.

[43] K. Kajantie, M. Laine, A. Rajantie, K. Rummukainen and M. Tsypin, JHEP 11 (1998) 011 hep-lat/9811004. 